\title{
Autologous Transplantation of Cryopreserved Ovarian Tissue to the Upper Extremity for the Preservation of Ovarian Function in Young Patient with Cervical Carcinoma
}

\section{Chen Meiyi ${ }^{1}$, Shao Yin ${ }^{1 *}$, Ma Liguo ${ }^{1}$, Zhuang Yongqing ${ }^{2}$, and Wang Xiaomei ${ }^{3}$}

${ }^{1}$ Department of Gynecology, Shenzhen People's Hospital, Second Affiliated Hospital of Jinan University, Shenzhen, P. R. China

${ }^{2}$ Department of Microsurgery, Shenzhen People's Hospital, Second Affiliated Hospital of Jinan University, Shenzhen, P. R. China

${ }^{3}$ The Engineering Lab of Synthetic Biology and the Key Lab of Biomedical Engineering, School of Medicine, Shenzhen University, Shenzhen, P. R. China

\begin{abstract}
For women of reproductive age with cervical carcinoma, they usually receive not only standard surgical treatment but also postoperative radiotherapy to increase the cure rates. However, infertility and premature ovarian failure are often occurred after radiotherapy. Autotransplantation of cryopreserved ovarian tissue is a new technique for preserving gonadal function for cervical cancer patients of reproductive age. Here, a successful ovarian function recovery through cryopreservation and autotransplantation in a case of 28-year-old patient with cervical cancer was reported. In brief, a slow-programmed freezing/thawing method was conducted for ovarian cortex cryopreservation. Six thawed ovarian cortical slices were transplanted to the left upper arm after the radiotherapy. The ovarian endocrine functions and follicle growth was successfully improved to normal level at 12 months after autotransplantation. In conclusion, autotransplantation of cryopreserved ovarian cortex for ovarian function preservation is a viable option for cervical cancer patients to gain the quality of life and avoid the suffering from conventional menopause hormone therapy.
\end{abstract}

Keywords: Ovarian cortex tissue; Cryopreservation; Autotransplantation; Ovarian function preservation

\section{Introduction}

Cervical carcinoma is the most common malignancy in women of reproductive age [1]. The high-dose chemotherapy and/or radiotherapy after surgery significantly raises the survival rate but causes deleterious effects on the ovarian tissue such as premature ovarian failure (POF), which dramatically attenuates the quality of life for cervical cancer patients who are willing to preserve fertility $[2,3]$. Fortunately, improvements in assisted reproductive technologies have led to remarkable progress in oncofertility and fertility preservation treatments [4]. Recently, cryopreservation of the ovarian for fertility preservation prior to cytotoxic therapies has been performed in cases of children and women suffering from various cancers [5]. Although cryopreservation of the ovarian is still considered experimental, it has been successfully applied in more than 86 patients for recovering of the ovarian function with the success rate of about $30 \%$ [6].

Two options of transplantation are available for ovarian function preservation in cervical cancer patients, including the orthotopic transplantation $[7,8]$ and heterotopic transplantation [9-11]. This study chose the heterotopic transplantation for ovarian tissue since it works better for preserving ovarian follicle integrity [12]. Ovarian tissue includes oocytes, follicle cells and stromal cells. Thus, the choice of cryoprotectant with maximum permeation capacity, minimum toxicity, and ice crystal formation potential is specific for each cell type. Three cryoprotectants are majorly used for cryopreservation so far such as propanediol, dimethyl sulphoxide (DMSO), and ethylene glycol $[13,14]$. Both Vitrification and slow-freezing have been reported for cryopreserving ovarian tissue [15]. Vitrification involves a rapid cooling time to avoid crystal formation and subsequent damage to ovarian tissue [4], however, it requires a higher concentration of cryoprotectants than that of slow-freezing, causing higher risk for cellular toxicity and osmotic trauma to ovarian tissue [16]. Thus far, only three live births have been reported after autotransplantation of vitrified ovarian tissue
$[17,18]$. Therefore, this study chose slow-freezing for cryopreservation of ovarian tissue.

In this study, we reported a successful experience of the ovarian function recovery after radiotherapy using slow freezing/thawing method for ovarian cortex cryopreservation in a 28 -year-old cervical cancer patient. Several parameters such as follicle development, level of serum estrogen $2\left(\mathrm{E}_{2}\right)$, follicle-stimulating hormone (FSH) and luteinizing hormone (LH) after transplantation were observed and recorded for evaluating the recovery of ovarian function in a follow-up of 21 months.

\section{Materials and Methods}

\section{Patient recruitment}

A 28-year-old woman with cervical cancer was referred to the Department of Gynecology at the Shenzhen People's Hospital in June 2013. Her weight was $49.5 \mathrm{~kg}$ and the blood pressure was 92/60 $\mathrm{mmHg}$ at the time of determination. Her medical history revealed 1 uncomplicated pregnancy. She had irregular post-coital bleeding without the use of oral contraceptives during the last year. During the last 2 months, she had symptoms of irregular vaginal blood loss and menstruation endless dripping. MRI examination showed a cervical

*Corresponding authors: Shao Yin, M.D, Department of Gynecology, Shenzhen People's Hospital, Second Affiliated Hospital of Jinan University No. 1017 Dong Min Bei Lu, Shenzhen, 518060, P. R. China, Tel: 075522948600; E-mail: doctorsy0755@163.com

Received March 28, 2017; Accepted April 10, 2017; Published April 17, 2017

Citation: Meiyi C, Yin S, Liguo M, Yongqing Z, Xiaomei W (2017) Autologous Transplantation of Cryopreserved Ovarian Tissue to the Upper Extremity for the Preservation of Ovarian Function in Young Patient with Cervical Carcinoma. J Cancer Sci Ther 9: 401-404. doi: 10.4172/1948-5956.1000449

Copyright: () 2017 Meiyi C, et al. This is an open-access article distributed under the terms of the Creative Commons Attribution License, which permits unrestricted use, distribution, and reproduction in any medium, provided the original author and source are credited. 
front lip tumor mass with an estimated dimension of $48 \mathrm{~mm} \times 37$ $\mathrm{mm} \times 26 \mathrm{~mm}$. No further abnormality was found in the uterus, ovaries, uterine attachment, ureter, liver or kidney. An examination of the tumor biopsy tissue specimen obtained from the patient confirmed the non-keratinizing squamous cell carcinoma in the cervix.

The surgery was performed on June 24,2014 . At a month after surgery, patient received radiotherapy for 25 times with the dosage of 2 Gy each time, which lasted for 5 weeks, from July 21, 2014 to August 24, 2014. At 9 month after surgery, the ovarian tissue autotransplantation was conducted on March 18, 2015. During surgical procedure, neither effusion in the abdominal cavity nor abnormalities were found on the lymph nodes in the pelvis or abdominal para-aortic. According to the International Federation of Gynecology and Obstetrics Stage, a radical hysterectomy with pelvic lymph node dissection was proposed to the patient with cervical cancer stage Ib2. Considering the adverse effect of postoperative irradiation and the age of the patient, she was counseled to undergo a surgical procedure to prevent gonadal damage through cryopreservation of the cortical ovarian tissue. The risk of limited experience with this technique was well-explained to the patient and the informed consent was obtained.

\section{Ovarian cortex extraction and cryopreservation}

The concept of this ovarian function recovery procedure was to transpose the right ovary to the upper abdomen and cryopreserve the left ovarian cortex followed by autotransplantation. Firstly, the left ovary was gently dissected from the surrounding tissue along with the transection of the utero-ovarian ligament through laparotomy. After oophorectomy, the ovarian cortical slices were ready to be processed. Before the freezing procedure, ovarian cortex was dissected from medulla and further cut into ultra-thin slices $(1 \mathrm{~cm} \times 1 \mathrm{~cm} \times 1 \mathrm{~mm})$. Moreover, frozen section biopsies were performed to make sure that the ovarian tissue was free of disease. In this report, we used TCM-199 medium (Hyclone, Logan, UT) combining with human serum albumin (Sigma, St. Louis, MO), DMSO (Sigma), and sucrose (Sigma) for equilibrating the ovarian cortical slices before the freezing procedure.

While the left ovary was removing from the pelvic region, the standard radical hysterectomy and pelvic lymphadenectomy were simultaneously performed. At the end of the procedure, the right ovary and fallopian tube were transposed to the upper abdomen by dissecting the ovarian vessels until the above umbilical level had been reached $(3 \mathrm{~cm} \sim 3.5 \mathrm{~cm})$. The position of the ovarian pedicle to the anterior abdominal wall was accomplished by suturing through the fascia tissue.

\section{Ovarian cortex cryopreservation}

The process of ovarian cortex cryopreservation was briefly described as follows: (1) Equilibrate slices of ovarian cortex for $30 \mathrm{~min}$ at $4^{\circ} \mathrm{C}$ in phenol-free TCM-199 medium with $1.5 \mathrm{~mol} / \mathrm{L}$ of DMSO, $5 \%$ human serum albumin and $0.1 \mathrm{~mol} / \mathrm{L}$ of sucrose, and place the vials on a tissue-roller during incubation to ensure even penetration of the cryoprotectant. (2) Load the tissue in cryovials into the automated freezing machine to cool from $0^{\circ} \mathrm{C}$ to $-7^{\circ} \mathrm{C}$ with a cooling rate of $-2^{\circ} \mathrm{C} /$ min. (3) Equilibrate for 10 minutes before manual seeding. (4) Keep cooling to $-40^{\circ} \mathrm{C}$ with a slower rate of $-0.3^{\circ} \mathrm{C} / \mathrm{min}$. (5) Keep cooling to $-140^{\circ} \mathrm{C}$ Math with a faster rate of $-10^{\circ} \mathrm{C} / \mathrm{min}$. (6) Transfer to liquid nitrogen dewar flask for storage.

\section{Thawing}

The thawing procedure must be executed precisely as follows: (1) Thaw at room temperature for 30 seconds. (2) Place in $37^{\circ} \mathrm{C}$ Math water bath for 3 minutes. (3) Wash tissues progressively in serial diluted DMSO cryoprotectant medium with $1 \%$ serum and $0.1 \mathrm{~mol} / \mathrm{L}$ of sucrose; gently agitate tissue for 5 minutes in each step $(1.0,0.5,0.25$, $0 \mathrm{~mol} / \mathrm{L}$ of DMSO). (4) Perform the last wash with medium containing $0.1 \mathrm{~mol} / \mathrm{L}$ of sucrose only. (5) Transfer to the fresh medium with serum, on ice.

\section{Results}

Pathology assessment of the tumor tissue specimen revealed a $5 \times 4 \times 3 \mathrm{~cm}^{3}$ squamous cell carcinoma of the cervix with infiltration depth $>1 / 2$ (Figure 1). The lymph nodes and ovaries were free of tumor metastases, but salpingitis was identified in the left fallopian tube. The parametrial tissue and vaginal wall were not affected. Considering the tumor diameter and the infiltration depth into the cervix, postoperative radiotherapy was executed as an adjuvant treatment. Symptoms of perimenopause were immediately exhibited in the patient after radiotherapy.

Six ovarian cortical slices were transplanted to the left upper arm at 6 months after radiotherapy (Figure 2). Postoperative Doppler ultrasound showed adequate blood flow in the transplanted ovarian cortical slices. No abnormality was observed in regular examinations. Ovarian follicle development was detected at 6 months after transplantation (Figure 3). The patient experienced cyclic swellings of the upper arm without serious discomfort and showed regular follicular activity at different stages through ultrasound monitoring. The ovary was slightly shrunk,
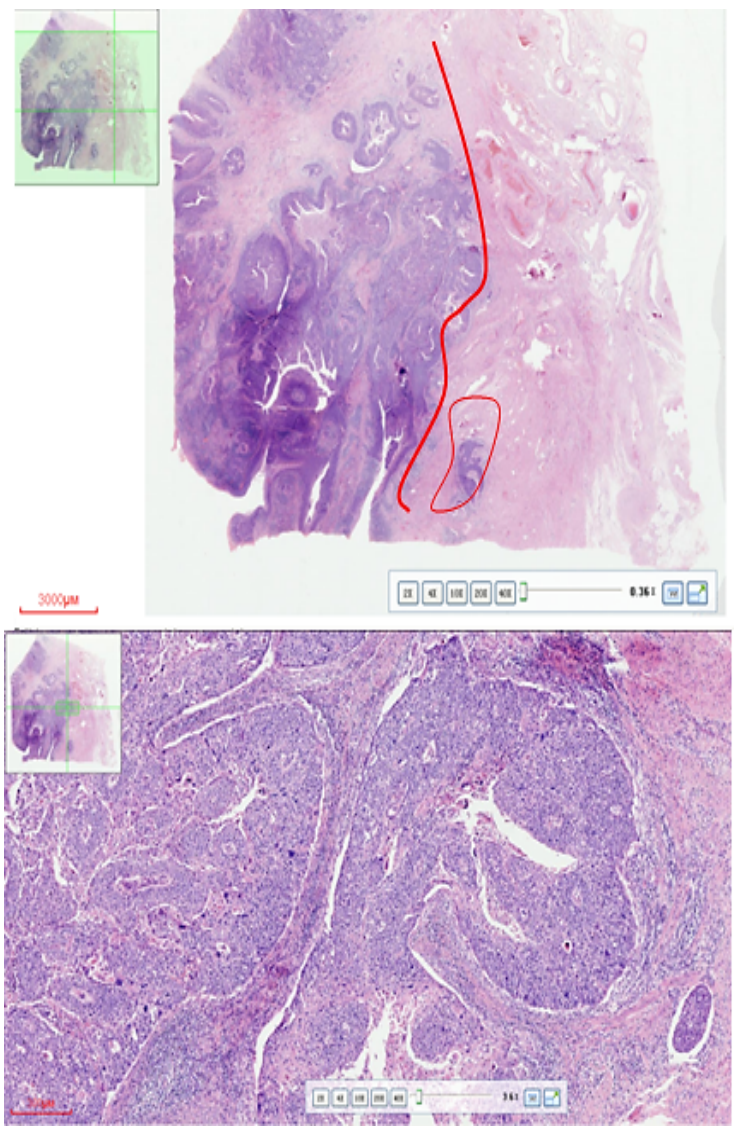

Figure 1: Pathology assessment of the tumor tissue. The tumor tissue specimen was observed and evaluated using H\&E staining. The red line separated the tumor tissue from the normal part, and revealed squamous cell carcinoma of the cervix with infiltration depth $>1 / 2$. 


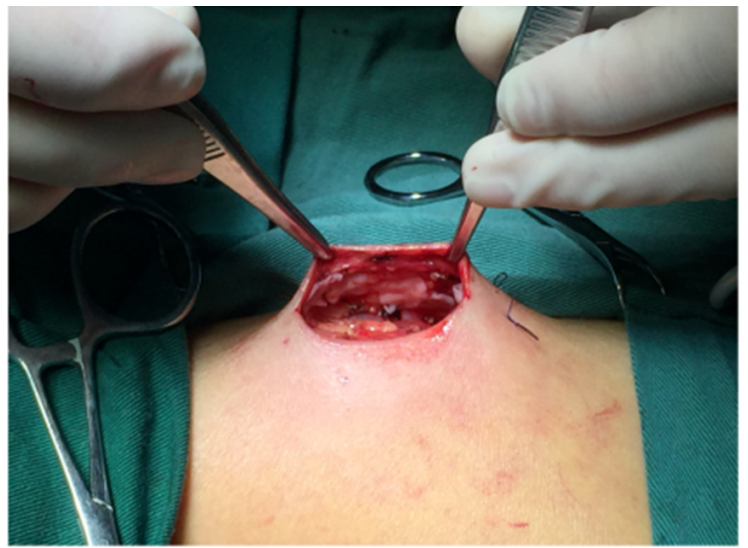

Figure 2: Transplantation of frozen-thawed autologous ovarian cortex tissue. Six thawed ovarian cortical slices were transplanted as a fan-shaped layer to the brachioradialis myofascial of the left upper arm.

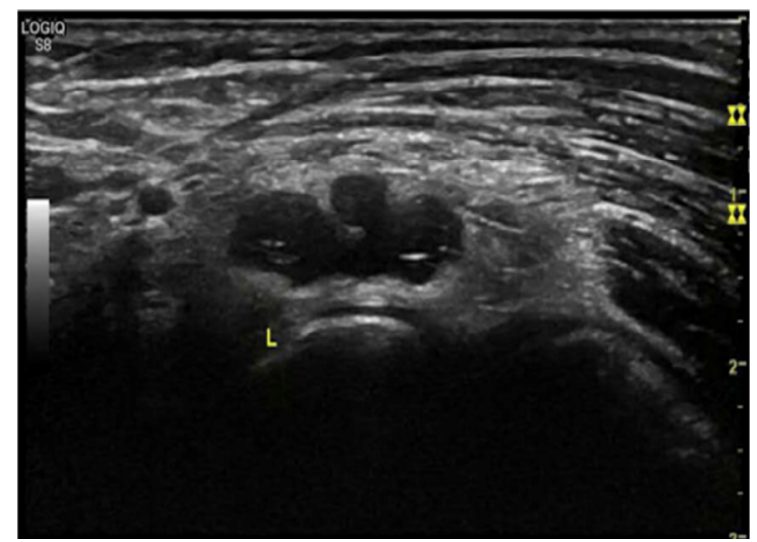

Figure 3: Follicle development in the subcutaneously grafted ovary. At 6 months after autotransplantation, several developing follicles were seen in the subcutaneously grafted ovary at the site of transplantation.

and the ovarian endocrine function was improved but no significant difference was detected at 6 months after surgery. At 9 months after transplantation, the serum $\mathrm{E}_{2}$ came back to normal level (264.47 pg/ $\mathrm{mL}$ ), and FSH and LH were deceased to non-menopausal levels (Figure 4). Furthermore, adequate ovarian endocrine functions such as vascularization and follicular activity were observed in the transplanted ovarian cortical pieces in a follow-up period of 21 months.

\section{Discussion}

Advances in the diagnosis and treatment of adult cancer have greatly prolonged the life expectancy in premenopausal women with cervical cancer. Unfortunately, the ovaries are very sensitive to cytotoxic treatment, especially to alkylating agents, generating higher risk for gonadal dysfunction [19]. In particular, ovarian toxicity is an important and common long-term side effect of radiotherapy or chemotherapy [20]. It has been stated that a dose of 5 to $20 \mathrm{~Gy}$ of radiotherapy administered to the ovary is sufficient to completely impair ovarian function, whatever the age of the patient [21]. Therefore, for the cervical cancer patients with urgent need of local radiotherapy, cryopreservation of ovarian tissue is one of the best alternatives to keep the fertility.

Radical hysterectomy with pelvic lymphadenectomy is the standard treatment for patients with early-stage cervical carcinoma.
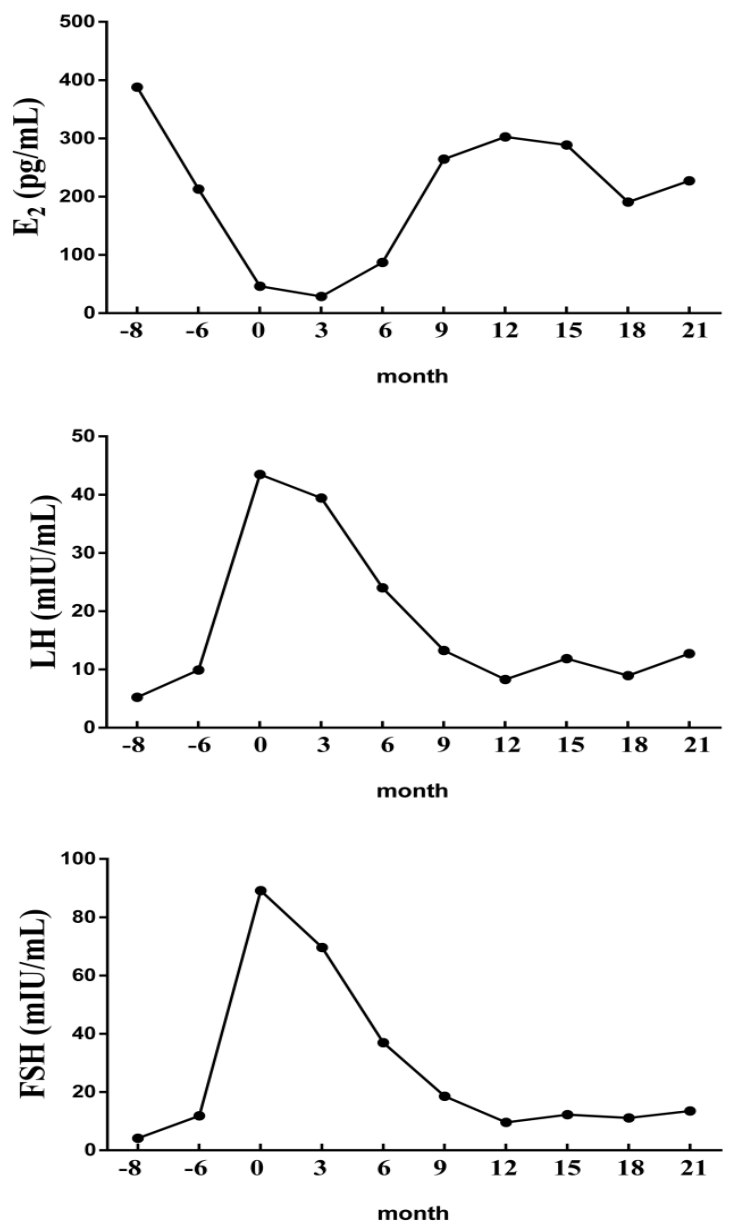

Figure 4: Cyclical hormonal production in the patient. Hormonal levels including E2, LH and FSH of the patient were monitored from 8 months before transplantation to 21 months after transplantation.

About $20 \%$ to $40 \%$ of patients would be suggested to receive this radical surgery followed by postoperative radiotherapy based on the standardized local tumor parameters such as lymph node metastases, parametrial involvement, affected surgical margins, and unfavorable local tumor factors $[16,17]$. Generally for a stage Ib2 cervical cancer patient underwent combined surgery and radiotherapy, the chances of tumor recurrence within 5 years is $9.3 \%$ and the survival rate is $90 \%$ according to the statistics in our institution, which is comparable to the calculation in other cervical cancer surgery study [22]. Radiotherapy has adverse effects on ovarian function, leading to ovarian atrophy and reduced follicle storage, and the degree and persistence of the damage are depended on the dose, irradiation field, and the patient's age [23]. Thus, preservation of ovarian function after treatment is of utmost importance for premenopausal cervical carcinoma patients.

Although transposing the ovaries outside of the irradiation field reduces the ovarian damage to approximately $5 \%$ to $10 \%$ in comparison with the untransposed ovaries [24-26], complications such as vasculature injury, fallopian tube infarction, and ovarian cyst formation are reported in several cases $[27,28]$. It is considered that cryopreservation and autotransplantation of ovarian tissue for fertility preservation is an experimental and pilot technique. The main aim of this strategy is to re-implant cortical ovarian tissue into the pelvic cavity or a heterotopic site, such as the forearm or abdominal wall. In this 
study, the perimenopause symptoms were immediately observed in the patient after radiotherapy. At 6 months after radiotherapy, the thawed ovarian cortical slices were placed in the forearm as the transplant recipient site. Ovarian endocrine function was established and follicle growth was observed. However, the patient refused to retrieve oocytes.

\section{Conclusion}

It has been shown that cryopreserving small non-vascularized cortical pieces of the ovary and implanting them as free tissue grafts in subcutaneous or muscle tissue outside the field of radiotherapy is a possible alternative for ovarian function preservation $[9,29]$. Nevertheless, transplantation of ovarian tissue pieces without a vascular pedicle requires the establishment of a new blood supply that takes 5 days [30]. Neovascularization from the surrounding tissue will take $\leq 5$ months before the initiation of follicular activity and hormone production [29]. This leads to a substantial loss of follicles in cryopreserved ovarian tissue, which is estimated to be at least $50 \%$ to $75 \%$ [31-33]. Ischemia and hypoxia, which are the critical factors in follicular loss, are the major limiting factors that have been shown to affect the longevity of the graft $[19,32]$. In this case, a follow-up period of transplanted ovarian cortical pieces for 21 months demonstrated adequate ovarian endocrine function including vascularization and follicular activity. Although a longer follow-up is still necessary for determining the long-term viability and endocrine function of the autotransplanted ovarian cortical pieces, these results revealed more insight into the longevity and functionality of the ovarian cortical tissue in the future. Taken together, cryopreservation of ovarian cortex tissue and autotransplantation are capable of preserving hormonal function and substantially improving patients' quality of life, which makes it a realistic way for preserving fertility for cervical cancer patients receiving high-dose pelvic radiotherapy.

\section{Acknowledgements}

This study was supported by the Basic Research Foundation of Shenzhen, grant no. JCYJ2013040292657781 and JCYJ20140416122811961.

\section{References}

1. Bloemers MC (2010) Preservation of ovarian function by ovarian transposition prior to concurrent chemotherapy and pelvic radiation for cervical cancer. A case report and review of the literature. Eur J Gynaecol Oncol 31: 194-197.

2. Meirow D (2000) Reproduction post-chemotherapy in young cancer patients. Mol Cell Endocrinol 169: 123-131.

3. Larsen P (2003) Reduced ovarian function in long-term survivors of radiationand chemotherapy-treated childhood cancer. J Clin Endocrinol Metab 88: 5307-5314.

4. Harada M, Osuga $Y(2016)$ Where are oncofertility and fertility preservation treatments heading in 2016? Future Oncol 12: 2313-2321.

5. Von WM, Stute P, Fluck C (2016) Autologous transplantation of cryopreserved ovarian tissue to induce puberty-the endocrinologists' view. Eur J Pediatr 175: 2007-2010.

6. Jadoul P (2017) Efficacy of ovarian tissue cryopreservation for fertility preservation: Lessons learned from 545 cases. Hum Reprod 32: 1046-1054

7. Donnez J (2006) Restoration of ovarian function after orthotopic (intraovarian and periovarian) transplantation of cryopreserved ovarian tissue in a woman treated by bone marrow transplantation for sickle cell anaemia: Case report. Hum Reprod 21: 183-188.

8. Von WM (2009) Cryopreservation and autotransplantation of human ovarian tissue prior to cytotoxic therapy: A technique in its infancy but already successful in fertility preservation. Eur J Cancer 45: 1547-1553.

9. Oktay K, Buyuk E, Rosenwaks Z, Rucinski J (2003) A technique for transplantation of ovarian cortical strips to the forearm. Fertil Steril 80: 193-198.

10. Oktay K (2005) Successful human ovarian autotransplantation to the upper arm. Cancer 103: 1982-1983.

11. Silber SJ (2012) Ovary cryopreservation and transplantation for fertility preservation. Mol Hum Reprod 18: 59-67.

12. Damasio LC (2016) Heterotopic ovarian transplantation results in less apoptosis than orthotopic transplantation in a minipig model. J Ovarian Res 9: 14

13. Youm HW (2014) Optimal Vitrification protocol for mouse ovarian tissue cryopreservation: Effect of cryoprotective agents and in vitro culture on vitrifiedwarmed ovarian tissue survival. Hum Reprod 29: 720-730.

14. Sheikhi M (2013) Preservation of human ovarian follicles within tissue frozen by Vitrification in a xeno-free closed system using only ethylene glycol as a permeating cryoprotectant. Fertil Steril 100: 170-171

15. Zhou XH (2016) Comparison of Vitrification and conventional slow freezing for cryopreservation of ovarian tissue with respect to the number of intact primordial follicles: A meta-analysis. Medicine (Baltimore) 95: e4095.

16. Pegg DE (2015) The role of Vitrification techniques of cryopreservation in reproductive medicine. Hum Fertil (Camb) 8: 231-239.

17. Salama M, Woodruff TK (2015) New advances in ovarian autotransplantation to restore fertility in cancer patients. Cancer Metastasis Rev 34: 807-822.

18. Donnez J, Dolmans MM (2015) Ovarian cortex transplantation: 60 Reported live births brings the success and worldwide expansion of the technique towards routine clinical practice. J Assist Reprod Genet 32: 1167-1170.

19. Donnez J (2006) Ovarian tissue cryopreservation and transplantation: A review. Hum Reprod Update 12: 519-535

20. Meirow D, Nugent $D$ (2001) The effects of radiotherapy and chemotherapy on female reproduction. Hum Reprod Update 7: 535-543.

21. Wallace WH (2005) Predicting age of ovarian failure after radiation to a field that includes the ovaries. Int J Radiat Oncol Biol Phys 62(3): 738-44.

22. Wang W (2016) Long-term oncological outcomes after laparoscopic versus abdominal radical hysterectomy in stage IA2 to IIA2 cervical cancer: A matched cohort study. Int J Gynecol Cancer 26: 1264-1273.

23. Meirow $D$ (2010) Toxicity of chemotherapy and radiation on female reproduction Clin Obstet Gynecol 53: 727-739.

24. Howell S, Shalet S (1998) Gonadal damage from chemotherapy and radiotherapy. Endocrinol Metab Clin North Am 27: 927-943.

25. Moawad NS (2017) Laparoscopic ovarian transposition before pelvic cancer treatment: Ovarian function and fertility preservation. J Minim Invasive Gynecol 24: $28-35$.

26. Takahashi N (2015) Five cases of cervical cancer where laparoscopic ovarian transposition was performed before pelvic radiotherapy. J Minim Invasive Gynecol 22: S203.

27. Morice P, Juncker L, Rey A, El-Hassan J, Haie-Meder C (2000) Ovarian transposition for patients with cervical carcinoma treated by radiosurgical combination. Fertil Steril 74: 743-748.

28. Pahisa J (2008) Laparoscopic ovarian transposition in patients with early cervical cancer. Int J Gynecol Cancer 18: 584-589.

29. Oktay K (2001) Endocrine function and oocyte retrieval after autologous transplantation of ovarian cortical strips to the forearm. JAMA 286: 1490-1493.

30. Donnez J, Dolmans MM (2010) Cryopreservation and transplantation of ovarian tissue. Clin Obstet Gynecol 53: 787-796.

31. Baird DT (1999) Long-term ovarian function in sheep after ovariectomy and transplantation of autografts stored at $-196^{\circ} \mathrm{C}$. Endocrinology 140 : $462-471$.

32. Hilders CG (2004) Successful human ovarian autotransplantation to the upper arm. Cancer 101: 2771-2778.

33. Nisolle M, Casanas-Roux F, Qu J, Motta P, Donnez J (2000) Histologic and ultrastructural evaluation of fresh and frozen-thawed human ovarian xenografts in nude mice. Fertil Steril 74: 122-129. 